\title{
SpecSyn: An Environment Supporting the Specify-Explore-Refine Paradigm for Hardware/Software System Design
}

\author{
Daniel D. Gajski, Fellow, IEEE, Frank Vahid, Member, IEEE, Sanjiv Narayan, and Jie Gong
}

\begin{abstract}
System-level design issues are gaining increasing attention, as behavioral synthesis tools and methodologies mature. We present the SpecSyn system-level design environment, which supports the new specify-explore-refine (SER) design paradigm. This three-step approach to design includes precise specification of system functionality, rapid exploration of numerous systemlevel design options, and refinement of the specification into one reflecting the chosen option. A system-level design option consists of an allocation of system components, such as standard and custom processors, memories, and buses, and a partitioning of functionality among those components. After refinement, the functionality assigned to each component can then be synthesized to hardware or compiled to software. We describe the issues and approaches for each part of the SpecSyn environment. The new paradigm and environment are expected to lead to a more than ten times reduction in design time, and our experiments support this expectation.
\end{abstract}

Index Terms - Embedded systems, estimation, exploration, hardware/software codesign, hierarchical modeling methodology, partitioning, refinement, specification, system design.

\section{INTRODUCTION}

$\mathbf{T}$ HE focus of design effort on higher levels of abstraction, driven by increasing system complexity and shorter design times, has led to the need for a system-level design methodology and supporting tools. To better understand the system design problem, we can isolate three distinct tasks. First, we must specify the system's functionality and constraints. Second, we must explore various system-level design alternatives, each consisting of an interconnection of system components and an assignment of functionality to them. System components include standard processors, custom application specific integrated circuit (ASIC) processors, memories,

Manuscript received March 17, 1995; revised July 3, 1996. This work was supported by the National Science Foundation under Grant MIP-8922851 and the Semiconductor Research Corporation under Grant 92-DJ-146.

D. D. Gajski is with the Department of Information and Computer Science, University of California, Irvine, CA 92664 USA.

F. Vahid is with the Department of Computer Science, University of California, Riverside, CA 92502 USA.

S. Narayan is with Ambit Design Systems, Santa Clara CA 95053 USA.

J. Gong was with the Semiconductor Systems Design Technology Group, Motorola, Inc., Tempe, AZ 85281 USA. She is now with Qualcomm, Inc., San Diego, CA 92121 USA.

Publisher Item Identifier S 1063-8210(98)01308-0. and buses. Third, we must refine the original specification into a new system-level description, which designers will use to create an implementation for each component.

In current practice, these three steps are carried out in an informal and ad hoc manner. Specifications are usually written informally in English or some other natural language. Exploration is done manually using mental or hand-calculated estimations of quality metrics such as performance, size, and power. The refined description is then created informally using block diagrams and English. Drawbacks of such informal techniques include the lack of early simulation, the lack of rapid feedback of quality metrics that result from design decisions, the lack of automated tools to explore more design alternatives while requiring less design time, and the lack of good documentation of each component's functionality as well as of the design decisions to aid in concurrent design, component integration and redesign.

The response in the research community to the above drawbacks has been to introduce simulatable specifications earlier into the design process, and to use automated tools to assist in the exploration of design alternatives. The specifyexplore-refine paradigm, which can also be thought of as a hierarchical modeling methodology, may further improve the situation. In such an approach, we first precisely specify the system's functionality, explore numerous system-level implementations with the aid of tools, and then automatically generate a refined description representing any implementation decisions.

More specifically, the following tasks, illustrated in Fig. 1, are necessary to create a system-level design.

- Specification Capture: To specify the desired system functionality, we decompose the functionality into pieces by creating a conceptual model of the system. We generate a description of this model in a language. We validate this description by simulation or verification techniques. The result of specification capture is a functional specification, which lacks any implementation detail.

- Exploration: We explore numerous design alternatives to find one that best satisfies our constraints. To do this, we transform the initial specification into one more suitable for implementation. We allocate a set of system components and specify their physical and performance constraints. We partition the functional specification among allocated components. For guidance in these exploration 


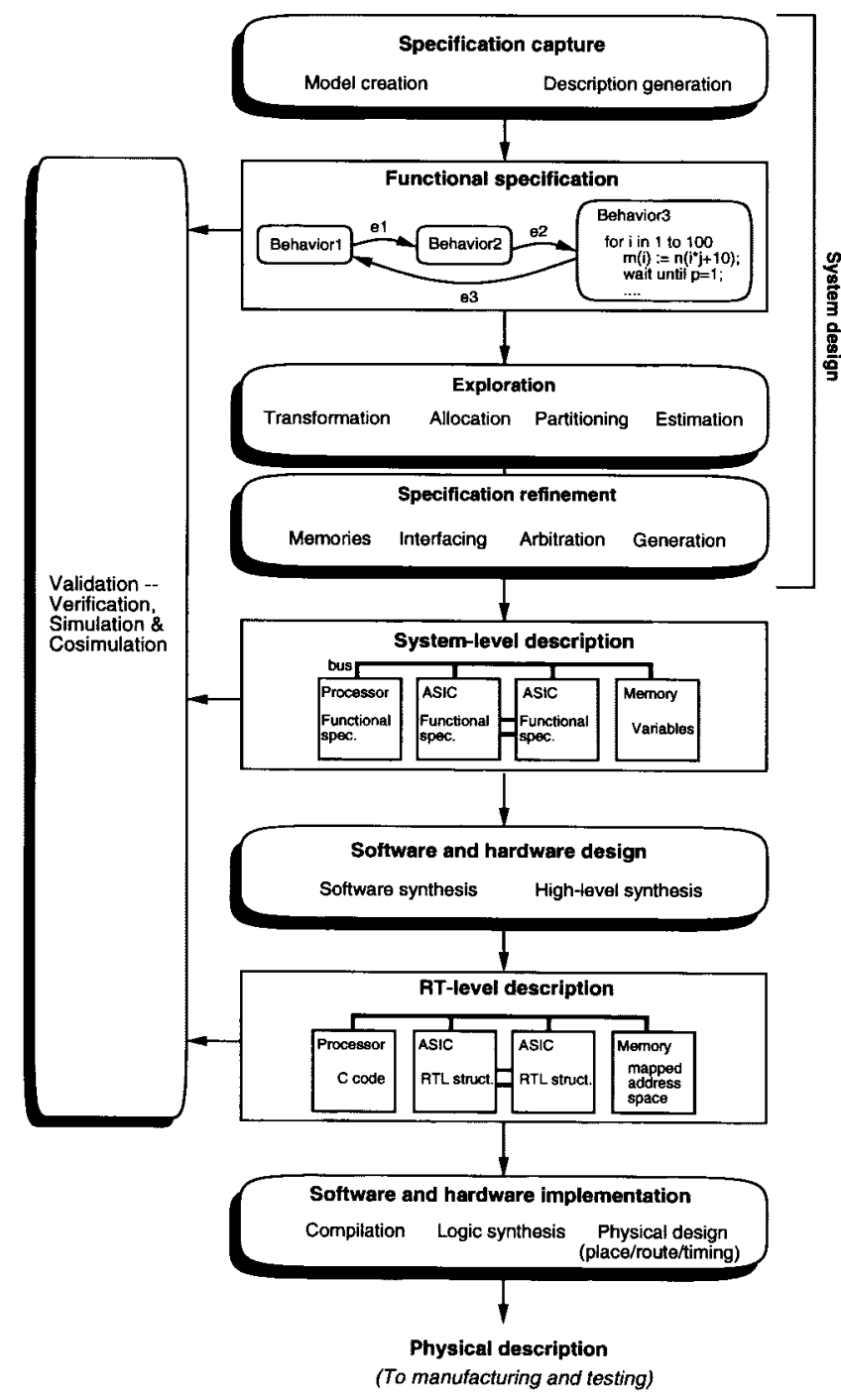

Fig. 1. The specify-explore-refine (SER) approach to system design.

subproblems, we estimate each alternative design's quality.

- Specification Refinement: We refine the initial specification into a new description reflecting the decisions that we have made during exploration. To do this, we move variables into memories, insert interface protocols between components, and add arbiters to linearize concurrent accesses to a single resource. Then, we generate a system description detailing the system's processors, memories, and buses and the functionality assigned to each. We use cosimulation to verify that this refined description is equivalent to the initial specification. The result of specification refinement is a system-level description, which possesses some implementation details of the system-level architecture we have developed, but otherwise is still largely functional.

Afterwards, we perform software and hardware design, where we create a design for each component, using software and hardware design techniques. A standard processor component requires software synthesis, which determines software execution order to satisfy resource and performance

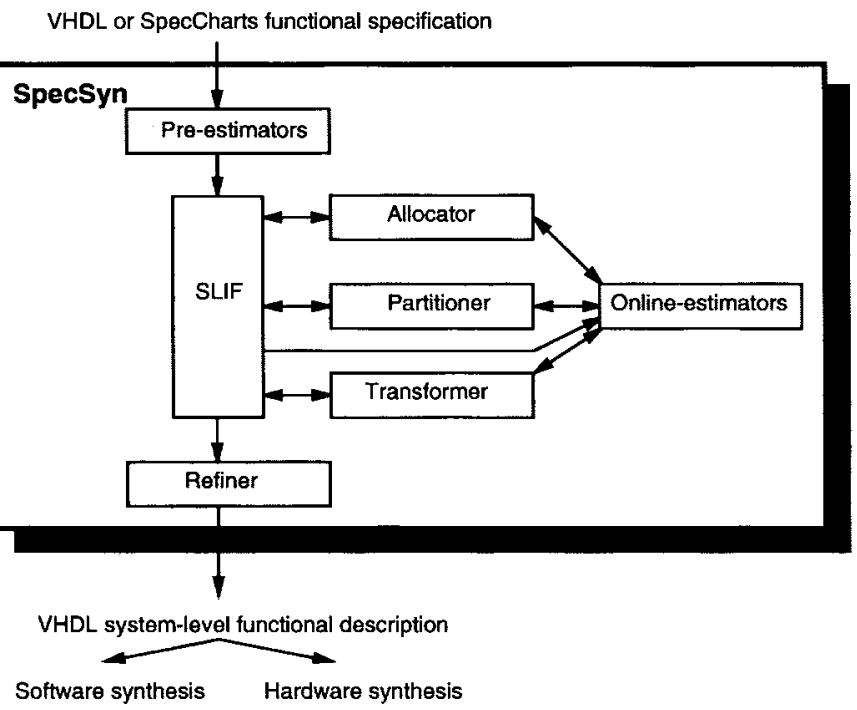

Fig. 2. The SpecSyn system-design environment.

constraints. We can obtain a custom processor's design through high-level (behavioral) synthesis [1], [2], which converts the behavioral description into a data path structure of registertransfer (RT) components from a library, such as arithmetic and logic units, registers, counters, register files and memories, along with a finite-state machine (FSM) controller that sequences the flow of data through the data path. The result of software and hardware design is an RT-level description, which may contain $\mathrm{C}$ code for each software component, and an FSM plus an RT-level netlist for each custom component. The RT-level description is then passed to software and hardware implementation for final implementation. Software components require compilation, while custom components require FSM and logic synthesis [3] followed by physical design, in which fine-grained digital components like gates or transistors are placed, routed and timed on an integrated circuit (IC).

We have developed the SpecSyn environment to support the specify, explore, and refine steps-the SER paradigm. The various parts of SpecSyn, illustrated in Fig. 2, correspond to the various system-design subtasks described above; each part will be discussed in detail in upcoming sections. Discussion of how SpecSyn differs from many related efforts is found in Section VI; however, we point out two key differences here. First, SpecSyn outputs a system-level description, which differs from the input only by the addition of system-level architectural features. This output can thus be treated as though it were hand-written. Specifically, it can be easily read and understood, used as documentation, input to simulators, input to behavioral synthesis, input to real-time schedulers (and ideally compilers), or designed manually. SpecSyn thus fits in well with current practice. Second, SpecSyn was developed as a general tool intended to support a wide variety of implementation component technologies, architectures, and heuristics, and new versions of such items can be added.

In this paper, we present an overview of the SpecSyn environment, discussing relevant issues, previous work, and 
solutions for each part. We then present industry experiments using SpecSyn and the specify-explore-refine paradigm.

\section{SPECIFICATION CAPTURE}

\section{A. Models and Languages}

Specifying a system's functionality is a difficult task, because the functionality is often complex and poorly understood. To ease the specification task, one decomposes the functionality into pieces according to some model, and captures that model in some language. This distinction between a model and a language is important, since the choice of a model affects the ease of the specification task much more than does the choice of a language. Common models include communicating sequential processes (CSP) [4], dataflow graphs, hierarchical FSM's, Petri-nets, and object-oriented models. Common languages include $\mathrm{C}, \mathrm{C}++$, VHDL, Verilog, Statecharts [5], and Java. Each language can capture many models, but certain languages excel for particular models. For example, Statecharts excels at capturing FSM's, even though VHDL and Verilog can also capture FSM's, albeit with more effort.

We observed that no existing model or language catered to the capture of embedded systems. Embedded systems are those systems whose functionality is determined mostly by interactions with the environment. Examples include most controller and telecommunication systems. We found that many such systems possess several characteristics, including state-transitions, exceptions, forking, and program-like computations, which are not all supported by any one existing model. State-transitions, exceptions and forking are supported by the hierarchical FSM model, while forking and program-like computations are supported by the CSP model. To overcome this lack of support, we developed the program-state machine (PSM) model, which is essentially a combination of hierarchical FSM's (Statecharts) and CSP. The model consists of a hierarchy of program-states. Each program-state can be decomposed into concurrent program-substates or sequential program-substates sequenced by arcs, as in Statecharts. However, unlike Statecharts, a third option is to decompose a program-state into sequential program statements. Because a program-state is not just a state but also a computation, two types of arcs are required: transition-on-completion (TOC), which is traversed when the computation has completed, and transition-immediately (TI), which is traversed when the arc event occurs, regardless of the computation stage. We also developed the SpecCharts language, which is an extension to VHDL, to capture the PSM model [6]. SpecCharts can be translated automatically to VHDL, which will be more complex than the original SpecCharts, but is simulatable and (ideally) synthesizable in a VHDL environment. Of course, the PSM model can also be captured directly in VHDL (with some additional effort); we are currently investigating techniques to capture the PSM model in Java and C++.

The choice of a language depends on more than just supported system characteristics, so SpecSyn accepts the industry standard of VHDL as input, as well as SpecCharts.
Though languages such as VHDL and Verilog lack support for certain embedded system characteristics, most notably for state-transitions, one can always use some more complex combination of other constructs, which of course is more timeconsuming and error-prone, but not impossible. For example, we can always capture state transitions using sequential program constructs. Such capture using less appropriate constructs is analogous to capturing a record using multiple scalar variables, capturing recursion using a stack, or capturing a parser using C's sequential constructs; all such captures are possible (and in fact support tools usually translate to such constructs during processing), but are tedious for humans to perform directly.

There are many other system characteristics that are not directly supported by languages such as SpecCharts, VHDL, Verilog, and C, including synchronous dataflow [7], queuing, complex timing constraints, and mixed analog/digital parts. No one language directly supports all characteristics, but hybrid models and languages that extend the number of supported characteristics, such as PSM and SpecCharts, seem to be a step in the right direction. For more information on PSM and SpecCharts, we refer the reader to [6] and [8].

In addition to specifying functionality, the designer must also specify design constraints. SpecSyn permits minimum and maximum constraints to be specified on behavior execution times and channel bit rates. Ideally, one would also be able to specify overall design constraints, such as power, board size, dollar cost, and design cost (if these items could be quantified). More specific design constraints, such as a component's size and I/O limitations, will be derived from each component's library entry later.

\section{B. Internal Representation}

The captured specification must be converted into an internal representation on which subsequent tools can operate. Representations commonly used for behavioral synthesis, including the control/dataflow graph (CDFG) and Value Trace [1], expose control and data dependencies between arithmeticlevel operations, which may be too fine-grained for system design tasks. Most good partitioning heuristics would require long run times on the resulting large numbers of objects, and estimators could not obtain meaningful preestimates (see Section III-C) for each object. Refinement into a readable system-level description also becomes a nearly impossible task. Thus, we chose to create a representation based on the coarser-granularity of procedural-level computations.

A second drawback of using behavioral synthesis representations stems from their focus on dependencies. Such dependencies are necessary for scheduling during behavioral synthesis, but are not essential to performing system design tasks. Representing dependencies between procedural-level objects requires us to replicate each object at each place that a procedure is called, since dependencies will differ for each call. This replication makes the system design task much more complex. Instead, we developed a representation that focuses on representing the accesses, rather than dependencies, among objects. 


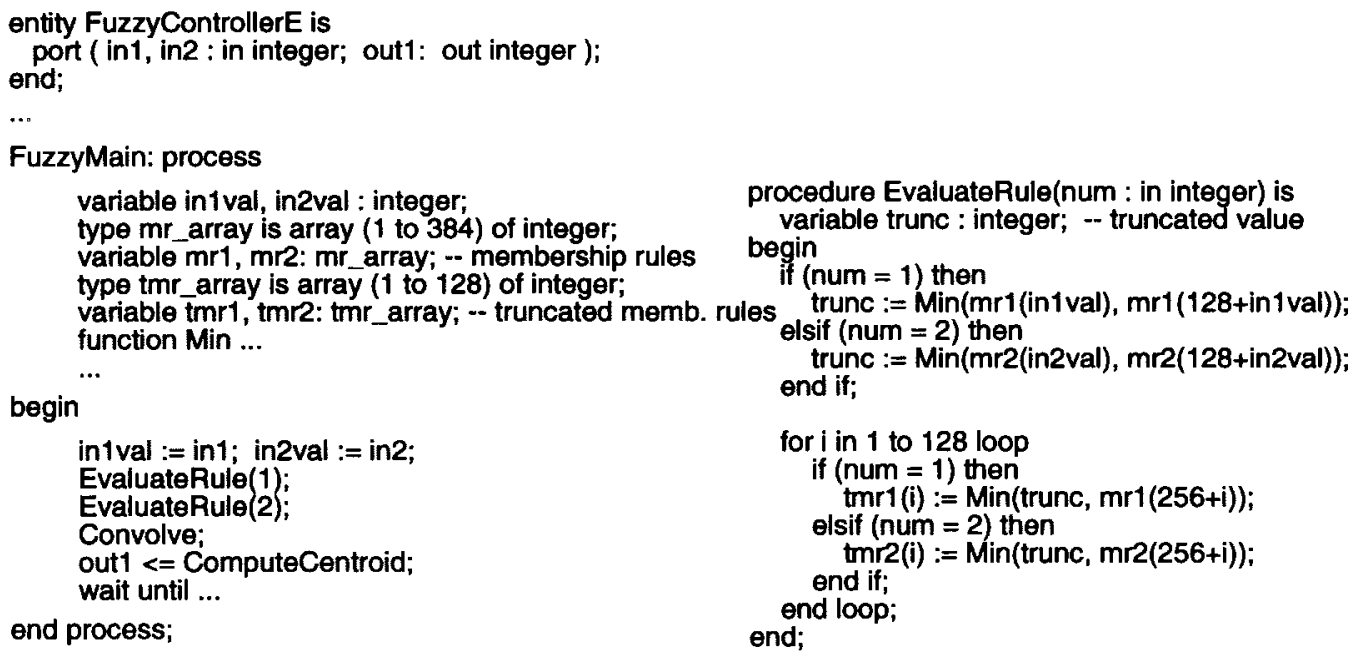

Fig. 3. Partial VHDL specification of a fuzzy-logic controller example.

For example, consider the partial VHDL specification of a fuzzy-logic controller in Fig. 3. Inputs $i n 1$ and $i n 2$ must be converted to output out 1 using fuzzy logic. The main process FuzzyMain first samples input values by writing them into variables in $1 \mathrm{val}$ and in2val. It then calls procedure EvalRule twice, once for each input, and that procedure fills an array ( $t m r 1$ or $t m r 2$ ) based on the input and on another predefined array ( $m r 1$ or $m r 2)$. After convolving the $t m r$ arrays, a centroid value is computed and output. The process repeats after a time interval.

We represent this specification as the directed graph in Fig. 4. Each graph node represents a behavior or a variable from the specification, where a behavior is a process or procedure, though for finer granularity we can consider statement blocks like loops by creating new procedures using a technique called exlining [9]. Each graph directed-edge represents a communication channel from the specification, where a channel represents a procedure call, a variable/port read or write, or a message pass specified using send/receive constructs. For example, process FuzzyMain, procedure EvalRule and variable in $1 \mathrm{val}$ are each represented by a node. The write of in $1 \mathrm{val}$ in FuzzyMain translates to a single edge, while the two calls of EvalRule by FuzzyMain translate to another single edge. Nodes representing processes are tagged to distinguish them from procedure nodes (hence the FuzzyMain node is shown in bold).

We refer to the representation as the Specification-level intermediate format (SLIF) since its granularity is that of behaviors and variables explicit in the specification. We refer to the part of SLIF shown so far as an access graph $(A G)$ since the relations between the behaviors/variables are defined by the accesses among those objects. The AG is similar to a procedure call-graph commonly used for software profiling, where an edge represents an access rather than a flow of data; the AG is more general since it also includes variables. Note that the AG uses only one node for EvalRule and one for Min, even though each behavior is called more than once with different dependencies for each call; thus, a large increase in the number of nodes is prevented using the AG. Sometimes

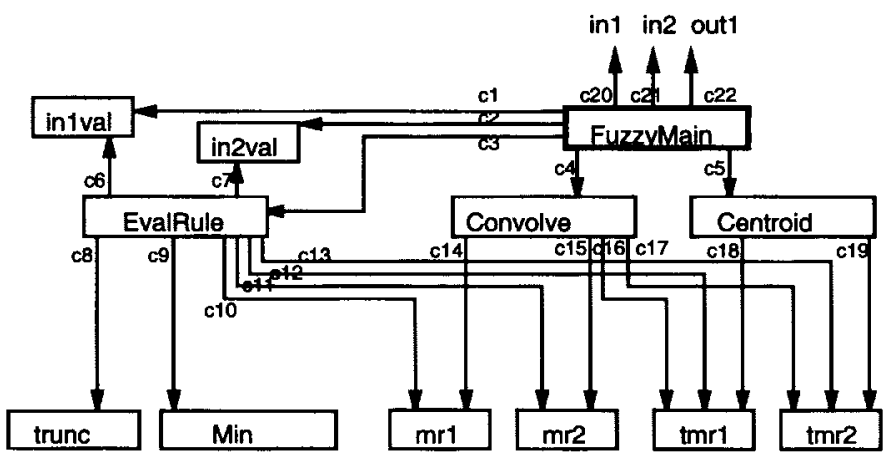

Fig. 4. Basic SLIF-AG for the example.

we do want multiple nodes, however, which can be handled using a procedure cloning transformation [10].

SLIF is annotated with numerous values, as shown in Figs. 5 and 6. We annotate each behavior and variable object with a list of size weights, one weight for each type of component to which the object may be assigned. For example, a variable object is annotated with the number of memory words required for storage in each library memory. A behavior is annotated with numbers of square microns, gates, and combinational-logic blocks for each custom chip, ASIC, and FPGA, respectively, on which the behavior could be implemented. [More complex annotations can be used to consider hardware sharing; see Section III-C3).] In addition, a behavior is annotated with the number of bytes for each possible standard processor.

We annotate each behavior and variable object with internal computation time (ict) weights for each possible component, corresponding to a variable's access time, or to a behavior's execution time excluding communication time. Times can be obtained with the aid of profiling and static estimation techniques [11]. We also annotate each edge with access frequency weights, which can also be obtained through profiling. Furthermore, we associate a bits weight with each edge, representing the number of bits sent during each transfer. For 


\begin{tabular}{l|rrrrrr} 
Object & ict_8051 & ict $\mathbf{X C 4 0 2 0}$ & lct_V100 & size_8051 & size_Xc4020 & size_v100 \\
\hline FuzzyMain & $\mathbf{5}$ & $\mathbf{8}$ & & 80 & 500 & \\
Intval & 0 & 0 & 0 & 2 & 80 & 2 \\
in2val & 0 & 0 & 0 & 2 & 80 & 2 \\
EvalRule & 778 & 522 & & 500 & 1600 & \\
Convolve & 800 & 600 & & 900 & 2000 & \\
Centrold & 2,500 & 2000 & & 700 & 4500 & \\
trunc & 0 & 0 & 0 & 2 & 80 & 2 \\
Min & 8 & 3 & & 30 & 850 & \\
mr1 & 0 & 0 & 0 & 768 & 30720 & 768 \\
mr2 & 0 & 0 & 0 & 768 & 30720 & 768 \\
tmr1 & 0 & 0 & 0 & 256 & 10240 & 256 \\
tmr2 & 0 & 0 & 0 & 256 & 10240 & 256
\end{tabular}

Fig. 5. SLIF behavior/variable annotations for the example.

\begin{tabular}{|c|c|c|c|}
\hline Object & accfreq & blts 8 rc & dst \\
\hline c1 & 1 & 6 FuzzyMain & in1val \\
\hline c2 & 1 & 6 FuzzyMain & in2val \\
\hline c3 & 2 & 8 FuzzyMain & EvalRule \\
\hline c4 & 1 & 0 FuzzyMain & Convolve \\
\hline c5 & 1 & o FuzzyMain & Centroid \\
\hline$c 6$ & 1 & 16 EvalRule & in 1val \\
\hline c7 & 1 & 16 EvalRule & in2val \\
\hline c8 & 129 & 16 EvalRule & trunc \\
\hline c9 & 129 & 32 EvalRule & Min \\
\hline$c 10$ & 65 & 32 EvalRule & $\mathrm{mr1}$ \\
\hline c11 & 65 & 32 EvalRule & $\mathrm{mr} 2$ \\
\hline c12 & 32 & 16 EvalRule & tmr1 \\
\hline $\mathrm{ct3}$ & 32 & 16 EvalRule & $\operatorname{tmr} 2$ \\
\hline
\end{tabular}

Fig. 6. SLIF channel annotations for the example.

each annotation, we might associate average, minimum and maximum values.

Annotations are computed during preestimation, and are combined into quality metric estimates during online estimation; Section III-C discusses these two estimation steps further.

\section{EXPLORATION}

Given a functional specification, we must proceed to create a system-level design of interconnected components, each component implementing a portion of that specification. A design's acceptability is evaluated by how well it satisfies constraints on design metrics, such as performance, size, power and cost. Since substantial time and effort are needed to evaluate a potential design, designers usually examine only a few potential designs, often those that they can evaluate quickly because of previous experience.

By using a machine-readable specification, we can automatically explore large numbers of potential designs rapidly. Exploration of potential designs can be decomposed into four interdependent subproblems: allocation, partitioning, transformation and estimation. We need not solve these problems in the given order; in fact, we will usually need to iterate many times before we are satisfied with our system-level design.

\section{A. Allocation}

Allocation is the task of adding components to the design. Many possible components exist. A standard processor is programmable and comes with widely used compilers and debuggers, but is usually slow or large. A special-purpose processor, such as a DMA controller or Fourier transformer, performs a specific function. A custom processor is synthesized to quickly execute a set of functions, but is harder to design and modify. An application-specific instruction-set processor (ASIP) is a programmable processor optimized for a particular class of applications, such as telecommunications. A memory stores variables. A bus implements communication between processors/memories.

The SpecSyn allocator permits allocation of any number of standard processors, custom processors, memories, and buses. Of course, allowing any allocation is only useful if the exploration tool understands the allocation; specifically, if the tool knows how to partition functionality among the components, knows how to estimate for such a partition, and can generate a refined description with behavior for each component. Incorporating such knowledge, especially that required for estimation, is very difficult, which is the reason that current tools only support a subset of possible allocations, such as a particular interconnection of a standard processor, memory, bus and custom processor [12], [13]. While SpecSyn permits a variety of allocations, its estimation models and heuristics must continually be improved to better apply to each.

Each component is characterized in a library by its constraints, and by a technology file. For example, a custom processor might be characterized by the maximum I/O pins and gates, and by a technology file describing an RT-component library. A standard processor is characterized by a maximum program memory size, a bus size, a maximum bus bitrate, and a technology file describing how to map a generic instruction set to the processor's instruction set [11]. A memory is characterized by the number of ports, number of words, word width, and access time. A bus is characterized by the number of wires, protocol, and maximum bit rates.

Ideally, we would also be able to allocate special-purpose processor components (e.g., DMA controllers), as well as hierarchical components, such as an ASIC which itself contains a standard processor core, memory, and several custom processor blocks.

Fig. 7 demonstrates an example allocation. StandardProc1 is an Intel 8051 with $4 \mathrm{~kb}$ of on-chip memory, and CustomProcl is a Xilinx XC4010 FPGA with $160 \mathrm{I} / \mathrm{O}$ pins and 10000 gates. Two $1 \mathrm{kB}$ memories are also allocated.

\section{B. Partitioning}

Given a functional specification and an allocation of system components, we need to partition the specification and assign each part to one of the allocated components. In fact, we can distinguish three types of functional objects that must be partitioned. One type is a variable, which stores data values. Variables in the specification must be assigned to memory components. The second is a behavior, which transforms data values. A behavior may consist of programming statements, such as assignment, if and loop statements, and it generates a new set of values for a subset of variables. Behaviors must be assigned to custom or standard processors. The third is the 


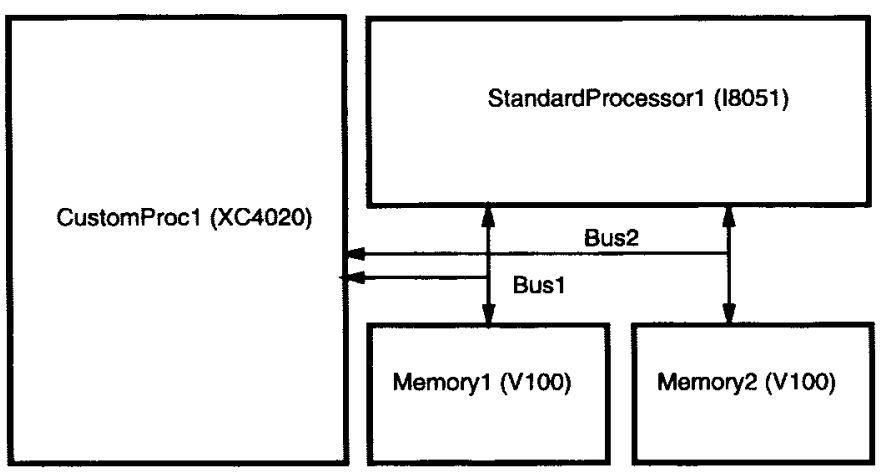

Fig. 7. An example allocation of components.

channel, which transfers data from one behavior to another. Channels must be assigned to buses. Specification partitioning strives to satisfy constraints, specified by the user as well as associated with allocated components.

1) Hardware and Hardware/Software Partitioning: A variety of techniques have evolved to assist the designer perform functional partitioning. We can consider two categories of techniques: hardware partitioning and hardware/software partitioning. The hardware partitioning techniques aim to partition functionality among hardware modules, such as among ASIC's or among blocks on an ASIC. Most such techniques partition at the granularity of arithmetic operations, differing in the partitioning heuristics employed. Clustering heuristics are used in [14] and [15], integer-linear programming in [16] and [17], manual partitioning in [18], and iterative-improvement heuristics in [19] and [20]. Other techniques for hardware partitioning operate at a higher level of granularity, such as in [21] where processes and subroutines are partitioned among ASIC's using clustering, iterative-improvement, and manual techniques. Experiments have shown tremendous advantages of functional partitioning over the current practice of structural partitioning [22].

Hardware/software partitioning techniques form the second functional partitioning category. These techniques focus on partitioning functionality among a hardware/software architecture. The techniques in [12], [13], [23], and [24]-[27] partition at the statement, statement sequence and subroutine/task levels, respectively.

In SpecSyn, both the hardware and hardware/software partitioning techniques are supported, since one can allocate any combination of hardware and software components and assign pieces of the specification to those components.

2) Heuristics: Instead of using one particular partitioning heuristic, SpecSyn uses a partitioning engine called GPP (general purpose partitioner). GPP is a library of functions with uniform interfaces, implementing the basic control strategies of numerous common heuristics, including clustering, group migration (an extension of Kernighan/Lin), simulated annealing, clique partitioning, genetic evolution, as well as custom heuristics. These control strategies are distinct from data structures and cost functions. A particular partitioning problem can be solved by calling a heuristic with the appropriate data structure and cost function-for example, circuit partitioning can be solved by passing a hypergraph data structure and a min-cut cost function. Each SpecSyn partitioning problem, including variables to memories, channels to buses, and behaviors to processors, is performed by passing the appropriate data structure and cost function and then applying the existing heuristics.

SpecSyn's approach to partitioning thus addresses the fact that heuristics, data structures, and cost functions are continually evolving. A new partitioning problem can initially be solved using a general heuristic. Then, once the problem definition has matured, one can develop and easily integrate a new custom heuristic. A user, after some experimentation, can choose the heuristic(s) with the appropriate result quality and runtime.

3) Manual Partitioning and Hints: We have also focused on supporting manual partitioning because of the importance placed on designer interaction. Such support not only involves providing the ability to manually relocate objects, but also allowing user control of the relative weights of various metrics in the cost function (see below), and automatically providing hints of what changes might yield improvements to the current partition. SpecSyn currently supports two types of hints. Closeness hints provide a list of object pairs, sorted by the closeness of the objects in each pair. Closeness is based on a weighted function of various closeness metrics. There are currently seven behavior closeness metrics supported [28]

- Connectivity is based on the number of wires shared between the sets of behaviors. Grouping behaviors that share wires should result in fewer pins.

- Communication is based on the number of bits of data transferred between the sets of behaviors, independent of the number of wires used to transfer the data. Grouping heavily communicating behaviors should result in better performance, due to decreased communication time.

- Hardware sharing is based on the estimated percentage of hardware that can be shared between the two sets of behaviors. Grouping behaviors that can share hardware should result in a smaller overall hardware size.

- Common accessors is based on the number of behaviors that access both sets of behaviors. Grouping such behaviors should result in fewer overall wires.

- Sequential execution is based on the ability to execute behaviors sequentially without loss in performance.

- Constrained communication is based on the amount of communication between the sets of behaviors that contributes to each performance constraint. Grouping such behaviors should help ensure that performance constraints are met.

- Balanced size is based on the size of the sets of behaviors. Grouping smaller behaviors should eventually lead to groups of balanced size.

There are also three closeness metrics supported for variables and for channels.

- Common accessors is based on the number of behaviors that access both sets of variables/channels. Grouping such variables/channels should result in fewer overall wires.

- Sequential access is based on the occurrence of sequential, rather than concurrent, access of the variables/channels by behaviors. Grouping sequentially 
accessed variables/channels into the same memory does not decrease performance, whereas grouping concurrently accessed ones might decrease performance due to access conflicts.

- Width similarity is based on the similarity of the variables'/channels' bit widths. Grouping variables/channels with similar bitwidths should result in fewer wasted memory/bus bits.

The other type of hint is called lookahead. Here, we generate all possible $n$ modifications of the current partition, where an $n$ modification is a sequence of $n$ moves of any objects from one group to another ( $n$ is user-defined). We again provide a list of such modifications, sorted by the partition improvement gained by each as measured by a cost function.

4) Cost Functions: Partitioning heuristics are guided by cost functions. A variety of cost functions can be supported. The following supported cost function focuses on satisfying constraints:

$$
\begin{aligned}
& \text { Costfct }=k_{1} \cdot F \text { (component1.size, } \\
& \text { component1.size_constr) } \\
& +k_{2} \cdot F \text { (component2.size, } \\
& \text { component2.size_constr) } \\
& +k_{3} \cdot F(\text { component1.IO, } \\
& \text { component1.IO_constr) } \\
& +k_{4} \cdot F(\text { behavior 1.exectime, } \\
& \text { behavior1.exectime_constr) ... }
\end{aligned}
$$

where the $k$ 's are user-provided constants indicating the relative importance of each metric, and $F$ is a function indicating the desirability of a metric's value. A common form of $F$ returns the degree of constraint violation, normalized such that zero indicates no violation, and one indicates very large violation. This form of $F$ causes the cost function to return zero when a partition meets all constraints, making the goal of partitioning to obtain a cost of zero.

The above cost function is very general, permitting us to satisfy constraints as well as to optimize certain metrics, without requiring specific knowledge in a heuristic of the constraints or optimization metrics. For example, if we wish to optimize execution time while satisfying size and I/O constraints, we can simply weigh size and I/O very heavily, so that violations of those constraints will not be tolerated. If we wish to focus first on just execution time, and then later on power, we can give the power constraint an initial weight of zero.

As an example of the results of partitioning, Fig. 8 shows a partition of several of the previous example's nodes among two memories, an ASIC, a processor and a bus. Note that four communication channels have been partitioned onto busl.

\section{Estimation}

Estimation of values for design quality metrics is required to determine if a particular system-level design (a partition of functions among allocated components) satisfies constraints, and to compare alternative designs. In this section, we describe

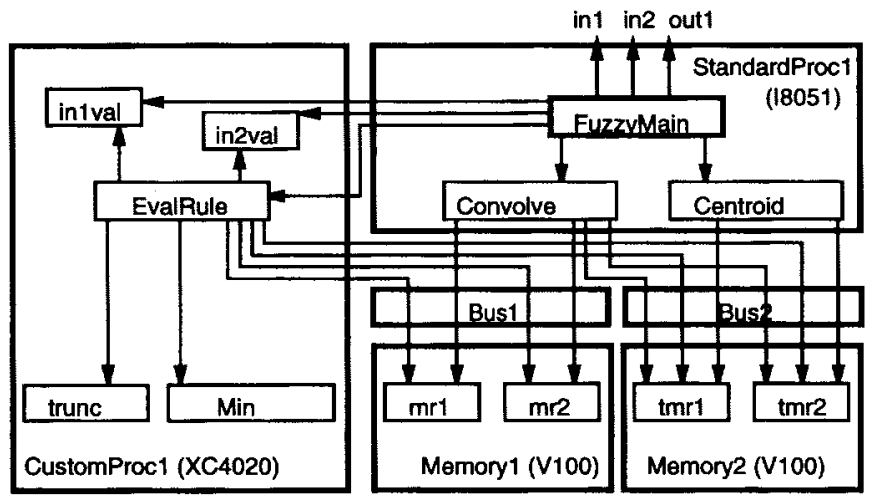

Fig. 8. Partitioning AG nodes among system components.

our two-level approach for fast and accurate estimation, and we provide details of our estimation models.

1) Preestimation and Online-Estimation: In general, more accurate estimates require more time, but time is very limited during exploration. (When comparing alternative options, fidelity is often more important than accuracy; see [8] and [29]). High accuracy can be achieved through synthesis, compilation, and simulation, i.e., by generating a refined description, creating an RT-level design using synthesis and compilation, measuring gates or bytes for size metrics, and performing simulations for performance metrics. However, the minutes or hours required by such an approach makes it unsuitable during exploration, when hundreds to tens of thousands of designs must be examined.

To decrease estimation time, an implementation model can be used, which is an implementation abstraction from which metric values can be derived, but which does not contain complete implementation details. SpecSyn uses a two-level technique to obtain metric values, as illustrated in Fig. 9.

1) Preestimation: Each functional object (behavior, variable and channel) is annotated with information (see Section II-B), such as the number of bytes for a behavior when compiled to a particular processor, the average frequency of channel access, or the number of channel bits. Preestimation occurs only once at the beginning of exploration, is independent of any particular partition and allocation, and may take seconds to minutes.

2) Online-estimation: Preestimated annotations are combined in complex expressions to obtain metric values for a particular partition and allocation. Online-estimation occurs hundreds or thousands of times during manual or automated exploration, so it must be completed in just milliseconds.

In most other approaches, exploration consists of only one level of estimation (or two levels where one is trivial), with another level coming only after RT-level design.

We now discuss SpecSyn estimation models for three metric types: performance, hardware size, and software size.

2) Performance: In SpecSyn's performance model, a behavior's execution time is calculated as the sum of the behavior's internal computation time (ict) and communication time. The ict is the execution time on a particular component, assuming all accessed behaviors and variables take zero time. 


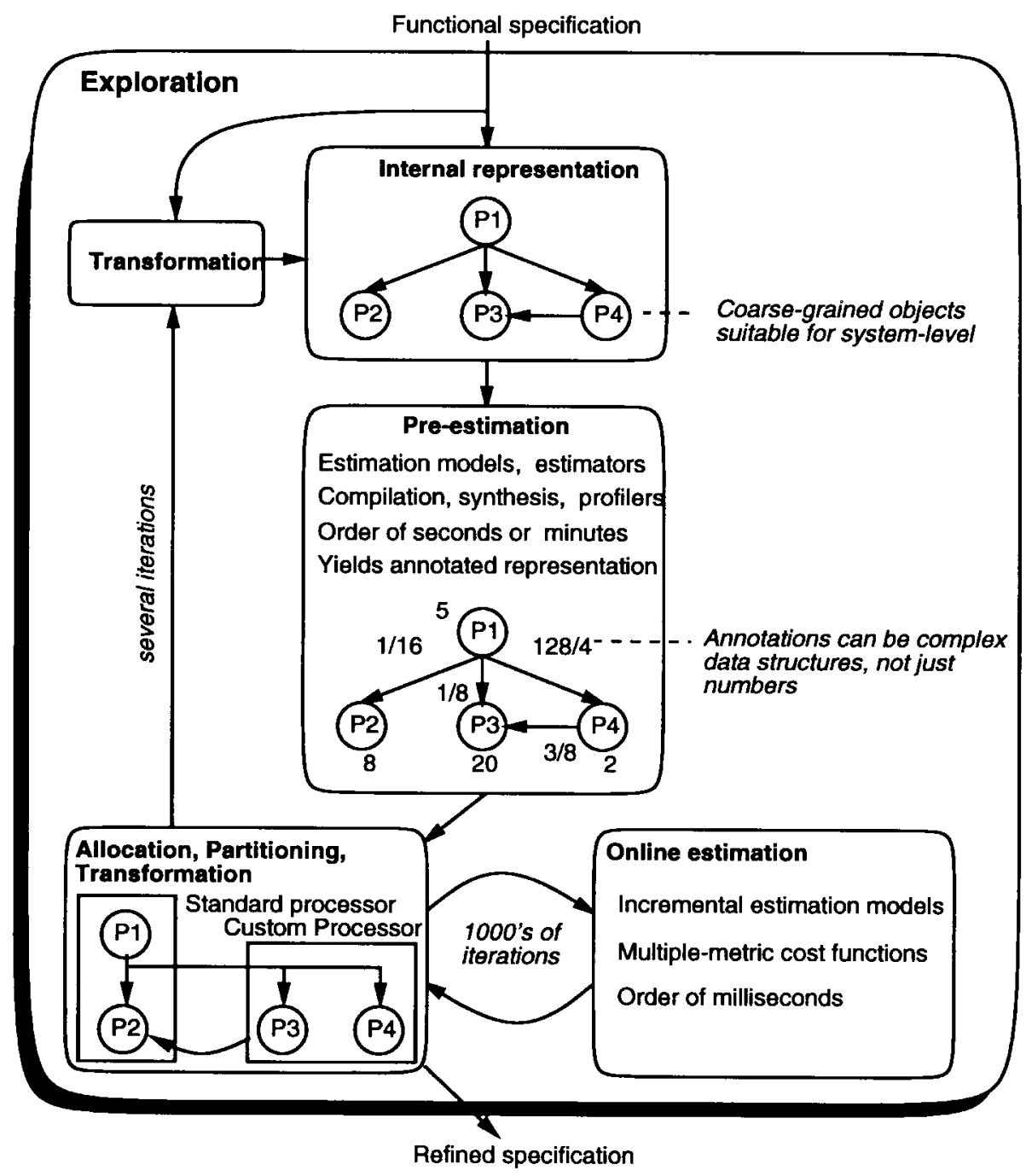

Fig. 9. Subtasks during exploration.

The communication time includes time to transfer data to/from accessed behaviors and variables, as well as the time for such accessed behaviors to execute (e.g., the time for a called procedure to execute and return). This model leads to some inaccuracy, since some computation and communication could occur in parallel, but the model seems to provide reasonable accuracy while enabling rapid estimations.

More precisely, execution time is computed as follows:

$$
\begin{aligned}
\text { b.exectime }= & \text { b.ict } p+\text { b.commtime } \\
\text { b.commtime }= & \sum_{c_{k} \in \text { b.outchannels }} c_{k} . \text { accfreq } \\
& \times\left(c_{k} . \text { ttime } \text { bus }+\left(c_{k} . \text { dst }\right) \text {.exectime }\right) \\
c_{k} . t \text { time } \text { bus }= & {\left[\text { bus.time } \times\left(c_{k} . \text { bits } \div \text { bus.width }\right)\right] } \\
\text { bus.time }= & \text { bus.timesame } \\
& \quad \operatorname{if}\left(c_{k} . \text { dst }\right) . p=p, \\
= & \text { bus.timediff } \text { otherwise. }
\end{aligned}
$$

In other words, a behavior's execution time equals its ict on the current component $\left(b . i c t_{p}\right)$, plus its communication time (b.commtime). The communication time equals the transfer time over a channel for each accessed object $\left(c_{k} . t t i m e_{b u s}\right)$, plus the execution time of each accessed object $\left(\left(c_{k} . d s t\right)\right.$.exectime $)$, times the number of such accesses $\left(c_{k}, a c c f r e q\right)$. The transfer time over a channel is determined from the bus data transfer time (bus.time) and the width of that bus (bus.width); if the data bits exceeds the bus width, then multiple transfers are used (as computed by the division). The bus_time is usually less when the communication is within one component.

Fig. 10 shows the execution-time equation for FuzzyMain of Fig. 4. For simplicity, the example uses fixed numbers for Convolve and Centroid communication times, whereas actually further equations should be used.

a) Preestimation: A behavior's internal computation time can be computed during preestimation through profiling and scheduling. Profiling determines the execution count of each basic block where a basic block is a sequence of statements not containing a branch. A schedule for each basic block is then estimated for each possible processor component, using compilation for standard processors and synthesis for custom processors. [Compilation techniques are discussed further in Section III-C4).] The summation over all blocks 


\begin{tabular}{|c|c|}
\hline FuzzyMain.et & $\begin{array}{l}=\text { FuzzyMain.ict } \\
+ \text { c1.accfreq * }(c 1 . t t+\text { in 1val.et }) \\
+ \text { c2.accfreq * (c2.tt }+ \text { in2val.et }) \\
+c 3 . a c c f r e q * \text { (c3.tt }+ \text { EvalRule.et }) \\
+c 4 . a c c f r e q * \text { (c4.tt + Convolve.et }) \\
+ \text { c5.accfreq * (c5.tt + Centroid.et })\end{array}$ \\
\hline inv1val.et & $=$ intval ict +0 \\
\hline inv2val.et & $=$ in 2 val ict +0 \\
\hline EvalRule.et & $\begin{aligned}= & \text { EvalRule.ict } \\
+ & \text { c8.accfreq * (c8.tt }+ \text { trunc.et }) \\
& \text { c9.accfreq * (c9.tt }+ \text { Min.et }) \\
& \text { c10.accfreq * }(c 10 . t t+m r 1 . e t) \\
& \text { c11.accfreq * (c11.tt }+ \text { mr2.et) } \\
& \text { c12.accfreq * (c12.tt }+ \text { tmr1.et }) \\
& \text { c13.accfreq * (c13.tt }+ \text { tmr2.et) }\end{aligned}$ \\
\hline Convolve.et & $=$ Convolve.ict +333 \\
\hline $\begin{array}{l}\text { Centroid } \\
\text { trunc }\end{array}$ & $\begin{array}{l}=\text { Centroid.ict }+454 \\
=\text { trunc ict }+0\end{array}$ \\
\hline Min & $=$ Min ict +0 \\
\hline $\mathrm{mr} 1$ & $=m r 1 i c t+0$ \\
\hline $\mathrm{mr} 2$ & $=m r 2 i c t+0$ \\
\hline $\operatorname{tmr} 1$ & $=\mathrm{tmr} 1 \mathrm{ict}+0$ \\
\hline $\operatorname{tmr} 2$ & $=t m r 2 i c t+0$ \\
\hline
\end{tabular}

Fig. 10. Execution-time equations for the example.

of each block's execution count times steps yields the total steps for the behavior. Multiplying by the step time, i.e., the clock period, yields an ict value. Note that processors using pipelining, caching or interrupts would require further refinements of the ict model. Each behavior is annotated with an ict value for each possible component.

Channel access frequencies are also determined through profiling. Any variable accesses or procedure call parameters can be encoded into bits as during synthesis. Bus times and widths are already associated with each bus.

Figs. 5 and 6 showed the annotations obtained during preestimation for the fuzzy-logic controller example.

b) Online estimation: Given a partition of every functional object to a component, the actual ict, bus values, and bus times become known. Thus, a behavior's execution time equation can be evaluated. When a partitioning heuristic moves an object, the object's ict value will change, and bus times may also change since objects previously on the same component will now be on different components, and possibly vice-versa. We only need to change those values and reevaluate the equation. In addition, any other equations that include the object's execution time must also be updated. If care is taken to maintain links from an object to all terms that change when the object is moved, then the updates can be done very quickly.

Fig. 11 shows the results of evaluating the execution time equations for the fuzzy controller example. Using the allocation and partition of Fig. 8, each object is assigned to a component (comp), each of which was bound to a library item (bind); based on this assignment, the current ict values are shown. Using these ict's, and the communication times based on the transfer times (not shown), the execution time (et) equations of Fig. 10 are evaluated. Thus, FuzzyMain executes in 8494 time units for the given allocation and partition.

3) Hardware Size: When several behaviors are assigned to a custom processor, we must estimate the size (e.g., number of

\begin{tabular}{l|llrrr} 
Object & comp & bind & let & et \\
\hline FuzzyMain & StdProc1 & 8051 & 5 & $\mathbf{8 , 4 9 4}$ \\
in1val & CustProc1 & XC4020 & 0 & 0 \\
in2val & CustProc1 & XC4020 & 0 & $\mathbf{0}$ \\
EvalRule & CustProc1 & XC4020 & 522 & $\mathbf{2 , 1 9 7}$ \\
Convolve & StdProc1 & 8051 & 800 & 1,133 \\
Centroid & StdProc1 & 8051 & 2500 & $\mathbf{2 , 9 5 4}$ \\
trunc & CustProc1 & XC4020 & 0 & 0 \\
Min & CustProc1 & XC4020 & 3 & 3 \\
mr1 & Mem1 & V100 & 0 & 0 \\
mr2 & Mem1 & V100 & 0 & 0 \\
tmr1 & Mem2 & V100 & 0 & 0 \\
tmr2 & Mem2 & V100 & 0 & 0
\end{tabular}

Fig. 11. Evaluating execution times for the example.

gates) required by that processor. The most accurate estimate is achieved by performing synthesis, but as discussed above, such an approach is too slow during exploration. Instead, some tools use a weight-based approach, in which preestimation consists of annotating each behavior and variable with a weight, and then a simple online-estimation sums the weights [12], [13]. Such an approach is fast, but may be inaccurate since it does not consider hardware sharing. Other research efforts [14], [15], [18], [30] use a design-based approach, in which an online-estimation roughly synthesizes a design for a given partition, omitting time-consuming synthesis tasks such as logic optimization. While more accurate, such estimators may require tens of seconds, which may be too slow for exploration of thousands of options.

SpecSyn uses an incremental update technique to achieve both the accuracy of design-based estimators and the speed of weight-based estimators. The technique takes advantage of the fact that many iterative-improvement partitioning heuristics, while exploring thousands of partitions, move only a few objects between one iteration and the next. Thus, using extensive information gathered during preestimation, we incrementally modify a custom processor's design in just milliseconds (constant-time).

SpecSyn uses a hardware design model similar to those in [8], [14], and [15], consisting of a control-unit/data path (CU/DP) as shown in Fig. 12. The CU/DP area can be computed as the sum of the following terms: Functional-unit (FU) size; Storage-unit size including registers, register files and memories; Multiplexer size; State-register size; Controllogic size; and Wiring-size. As shown in Fig. 13, each term is a function of basic parameters, including the number of possible states, the number of control lines, the number of states each control line is active, the number of bits and words for each storage unit, the number of bits and type of each functional unit, the number of sources of each storageunit input, functional-unit input, and data path output, the number of data path connections, and the number of data path components. For example functions, see [31].

a) Preestimation: The parameters are computed for each functional object during preestimation, by performing rough synthesis on each object. Each object is then annotated with the computed parameters. Such computation can take seconds or 


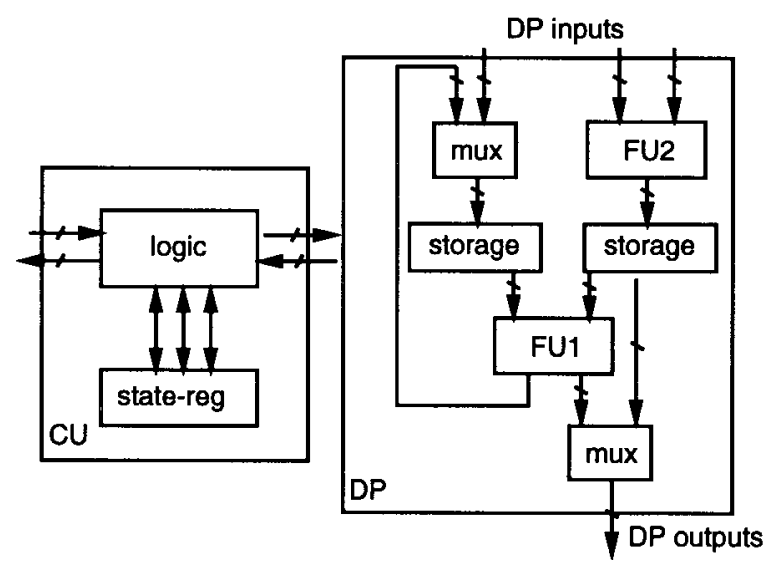

Fig. 12. CU/DP area model.

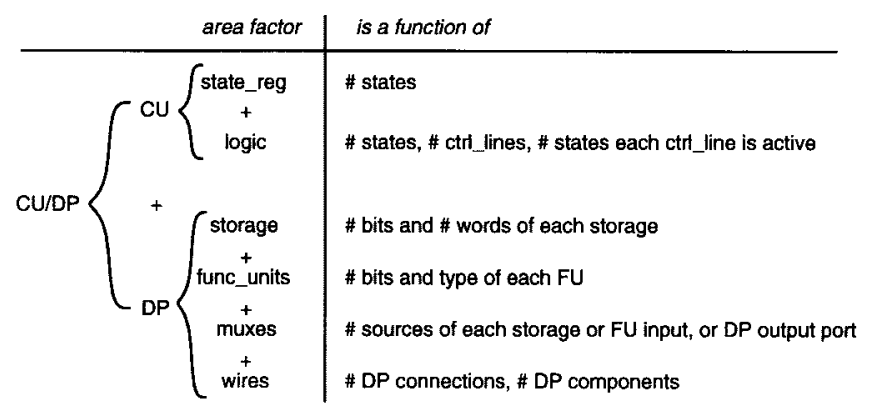

Fig. 13. Equation and terms for computing CU/DP area.

minutes. Given an initial partition of functional objects among custom processors, we can obtain a rough design of each processor by intelligently combining its objects' parameter annotations. For example, we can determine the number of possible custom processor states $S$ by summing the objects' possible states (in our model, a custom processor implements sequential objects from one process; multiple processes would require multiple processors) and then creating a state register of size $\log (S)$ bits. As another example, we can determine the number of FU's by taking the union of the objects' FU's (since sequential behaviors can share FU's). Note that the terms, such as state register size and number of FU's, are not obtained by simple addition; in fact, terms may actually be nonlinear with respect to the parameters. See [31] for details on computing all the terms from the objects' parameters.

b) Online estimation: When a partitioning heuristic removes an object from a processor, we update that processor's terms. Some terms can be updated simply by examining the object's annotations. For example, the number of possible processor states is reduced by the object's number, and the state register size recomputed using the log function. On the other hand, other terms require further examination. For example, an object might require a particular FU, but removing that object only removes that FU if no other object uses the FU; thus, we keep track of which objects use each FU. Likewise, removing an object might not eliminate a multiplexor, but might reduce its size since certain sources are no longer needed; thus, we keep track of which objects require each source. Updating a processor's design for removal of an object, as well as the complementary action of adding an object, can be done in constant time [31].

Note that we can merge the information from the functional objects because of their coarse granularity; otherwise, the ignored interobject effects would result in poor accuracy.

4) Software Size: A straightforward model of a processor's software size is that of the summation of the processor's functional objects' sizes. While neglecting interprocedural optimization, such optimization is likely not large, so this model yields fairly accurate estimates.

a) Preestimation: Ideally, we could determine a functional object's size by simply compiling the object for each possible target standard processor, as shown in Fig. 14(a). Unless the target processor is the same as the host machine processor on which SpecSyn is running, such compilation will require a cross-compiler, i.e., a compiler that runs on one processor but generates code for another. However, a cross-compiler may not be available on the host machine. For example, suppose the host machine is a Sparc and the target processor an Intel 8051. We probably do not have an 8051 compiler that runs on the Sparc; instead, we probably have one that runs on an $\mathrm{x} 86$ processor.

SpecSyn supports a method for estimating software size even when a cross-compiler is not available. The method uses a generic processor model and a single compiler, as shown in Fig. 14(b). A functional object's size is first compiled into generic three-address instructions. Using available processorspecific technology files listing the number of bytes that each generic instruction would require in each processor, the estimator computes the software size. A target processor's technology file can be developed based on the size information of the processor's instruction set; note that developing such a file is substantially simpler than developing a back-end compiler. Details on deriving technology files for specific processors are given in [11].

Note that the same generic processor approach would be applied for software performance estimation. Specifically, the technology file of the target processor would include not only the bytes but also the number of steps for each generic instruction.

Some experiments comparing the generic model with the processor-specific model yielded inaccuracy of roughly $7 \%$ [11].

b) Online estimation: Online software size estimation consists simply of increasing or decreasing the processor size by the size of the added or removed functional object.

\section{Transformations}

A functional specification serves the purpose of precisely defining a system's intended behavior. Such a specification usually will be read by humans as well as input to synthesis tools. Unfortunately, a specification written for readability may not directly lead to the best synthesized design. As a result, designers often try to juggle synthesis considerations with readability considerations while writing the initial functional specification. Such juggling usually leads to lower readability, less portability, and more functional errors; hence, many of 


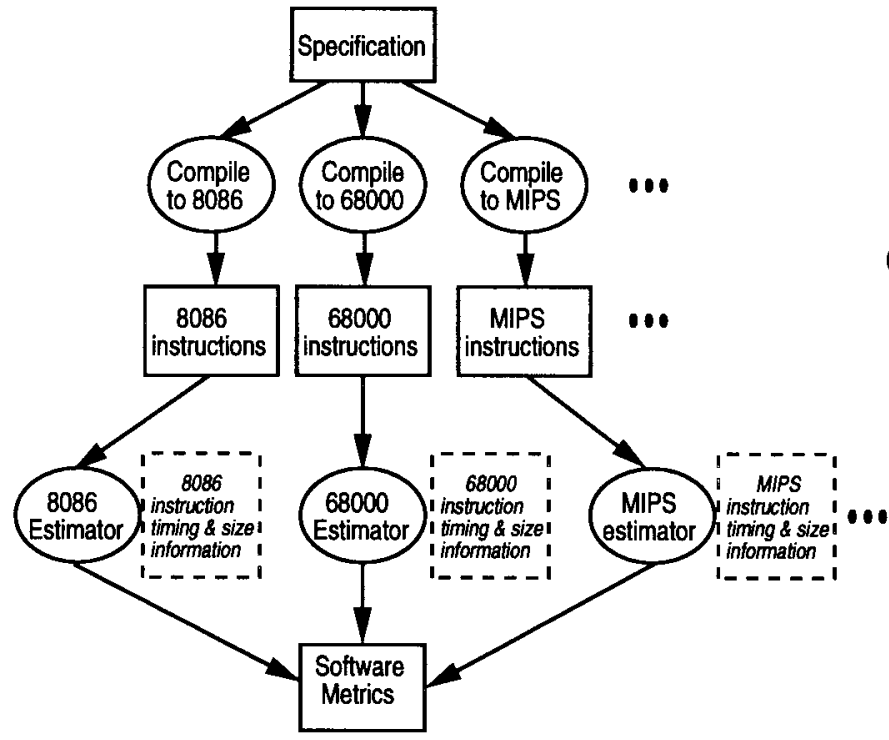

(a)

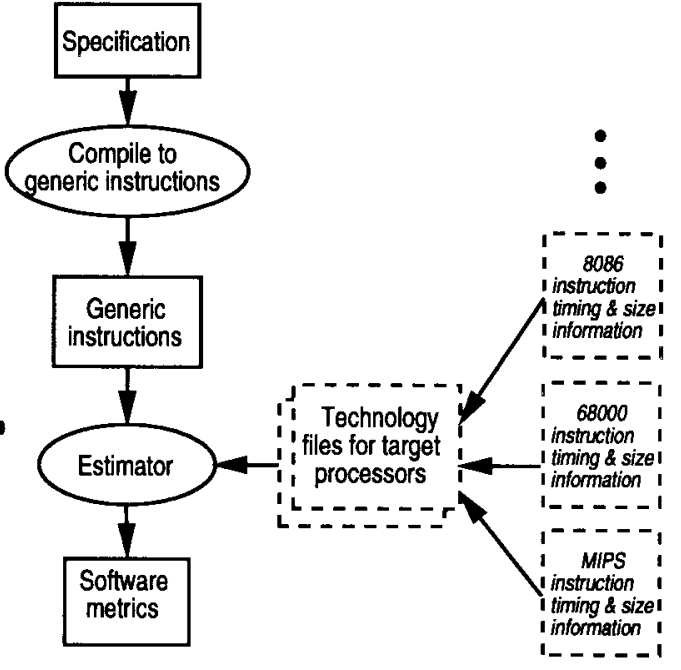

(b)

Fig. 14. Software size estimation: (a) processor-specific model and (b) generic model.

the advantages of a top-down approach are greatly diminished, ultimately leading to longer design times.

To solve this problem, SpecSyn provides a suite of automated transformations. As shown in Fig. 9, transformations can be applied on the SLIF or on the specification. SLIF transformations occur in an "inner loop" along with allocation, partitioning and online estimation, being applied thousands of times. Specification transformations occur in an "outer loop," which is followed by rebuilding of the SLIF and reannotation.

One specification transformation is procedure exlining [9]. Exlining is the inverse of procedure inlining; namely, replacing sequences of statements by procedure calls. Since procedures determine SLIF granularity, exlining is a means for achieving finer-granularity. There are two types of exlining. Redundancy exlining seeks to find and replace redundant statement sequences. Distinct-computation exlining seeks to break a large procedure into several smaller procedures, even though each may only be called once. Redundancy exlining is a very hard problem; presently, we encode each statement into a character string indicating the statement type, symbolic target and sources, concatenate each such string into one large one, and then use the agrep approximate pattern matching tool to find potential redundancies. Not all matches found by such an approach are necessarily redundancies, so user interaction is required. Distinct-computation exlining is in fact very similar to the problem addressed in [15]. Statements can be clustered together based on a number of closeness metrics. Simulated annealing can be used to further improve the statement clusterings.

A second specification transformation is procedure inlining, which achieves coarser granularity and distributes computations among calling behaviors, eliminating potential computation bottlenecks. Other possible transformations include process merging [32], where two processes are sequentialized into one to reduce hardware size, and process splitting [33], where one process is split into two concurrent ones. We plan to investigate such process transformations. A variety of other optimizing transformations with origins in software compilation could also be applied [34], [35].

Turning to SLIF transformations, preclustering [28] merges nodes that should probably never be separated, thus achieving coarser granularity. Procedure cloning [10] duplicates procedure nodes so that each calling behavior has its own copy, without necessarily inlining that copy; such cloning is analogous to logic duplication during logic-level partitioning. Port calling [36] inserts a node for sending or receiving data to external input/output ports; such nodes enable better distribution of $\mathrm{I} / \mathrm{O}$ among components, similar in idea to parallel I/O chips.

\section{REFINEMENT}

Refinement is the generation of a new specification for each system component after exploration has yielded a suitable allocation and partition. The refined specification should be both readable and simulatable, enabling further verification and synthesis. We now describe specification refinement tasks required after system design.

\section{A. Interfacing}

An important task is interface generation. Abstract communication channels were assigned to physical buses. Interface refinement determines the buswidth and the protocol for the bus that will implement the channels. A bus (such as a PC ISA bus) may already have these items fixed, in which case they are simply looked up. Alternatively, a bus may be flexible, in which case the best width and protocol must still be determined; algorithms and techniques have been reported in [37], [38]. After determining the protocol to meet design constraints, structure can be created for the protocol using techniques in [39]-[41]. 


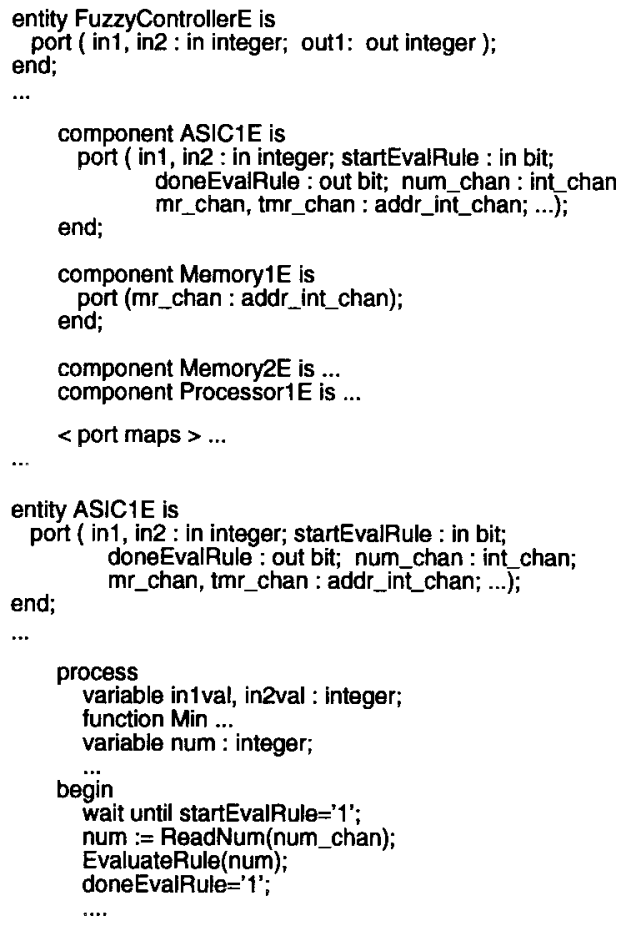

Fig. 15. Refined fuzzy-logic controller VHDL partial specification.

\section{B. Memories}

Another task is memory refinement associated with the implementation of variables assigned to memories. The variable accesses must be replaced by references to the corresponding memory locations.

\section{Arbitration}

A third task, arbiter generation, inserts an arbiter behavior where there is a resource contention, i.e., where two behaviors could access the same memory or bus simultaneously.

Note that, while during partitioning we abstracted communication implementation to the problem of mapping channels to buses, during refinement we must now deal with more complex communication issues involving protocols and arbitration. Such complex communication results in new behaviors (protocols and arbiters), which may later be synthesized, or possibly mapped to existing communication components like serial communication controllers or direct-memory-access controllers.

\section{Generation}

The final task of refinement is the actual generation of a refined description. The new description should be readable, modifiable, simulatable, and synthesizable. We use the following technique to generate a refined description. First, we create a VHDL entity for each system component. Second, for each behavior that represented a process in the original specification, we create a VHDL process inside the component to which the behavior has been assigned. Third, we describe activation for separated behaviors, i.e., those behaviors that have been assigned to a component different from their calling behaviors. The simplest approach to achieving such activation would be to create a single process for each such behavior,

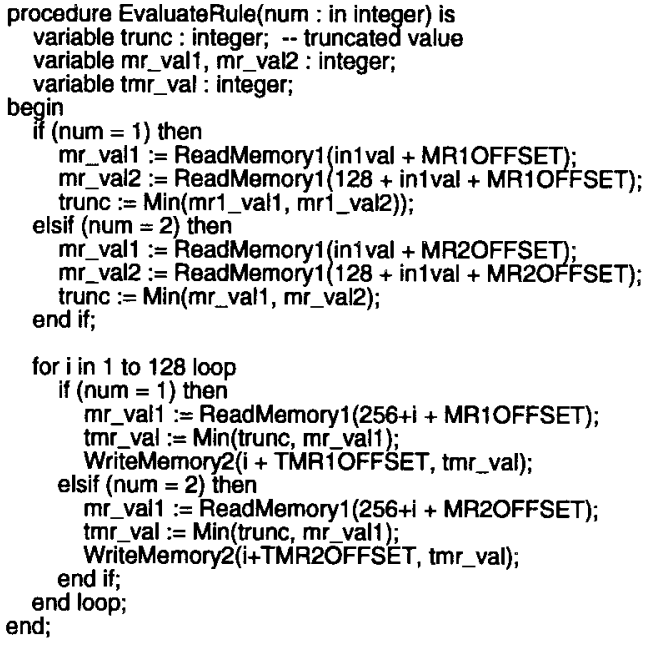

where the process would wait until it was activated via a control signal, would execute the behavior, and then would indicate completion via another control signal. However, such an approach results in an excessive number of processes (one for each separated behavior) and control signals. A better approach is to combine all separated behaviors that we know to be sequential (i.e., all those behaviors that belong to the same process), and that have been assigned to the same component, into a single process. This process would wait until it was activated, would execute one of its behaviors based on a newly introduced mode signal, and would indicate its completion. Fourth, we insert communication protocols and arbiters, as described above. We use VHDL send/receive procedures to hide the protocol details, and use additional VHDL processes to describe the arbiters.

\section{E. Validation}

To verify the system-design decisions, we can simulate the refined specification. When certain components use different models of computation than other components or contain different levels of details, different simulation approaches must be combined to obtain a simulation of the complete system. Such combination is called cosimulation. A variety of approaches to cosimulation are described in the literature, such as in [42]-[45]. The refined specification can serve as input to most of these approaches.

In Fig. 15, we show a refined specification for the system design shown in Fig. 8. Due to space limitations, the figure shows only a part of the refined specification. The interface of the fuzzy controller remains unchanged. However, its contents now consist of many more details than in the original specification of Fig. 3. For example, the top-level view of the controller now consists of instantiations of an ASIC, two 
memories, and a processor component, along with the interconnections among those components. The ASIC component, in turn, is defined as an entity with several ports. The first two ports, in 1 and in 2 , simply connect with the external inputs with the same names. The next two ports, startEvalRule and doneEvalRule, would be used by the FuzzyMain process on the processor to activate the EvalRule procedure on the ASIC. The last three ports shown, num_chan, mr_chan and tmr_chan, are composite data types that describe the signals necessary for fetching the num parameter from FuzzyMain, for fetching $m r 1$ and $m r 2$ data from Memoryl, and for storing tmr 1 and tmr2 data to Memory2. The ASIC's behavior consists of a single process, which waits for an activation signal, fetches the num parameter, and calls EvalRule with that parameter.

EvalRule is a procedure found in this process, identical to the procedure in Fig. 3, except that the $m r$ and $t m r$ arrays can no longer be accessed as global variables. Instead, they must be accessed using new subroutines that read data from Memoryl and write data to Memory2. Those subroutines describe the detailed communication protocol for such memory accesses, and would usually be found in a communication-protocol VHDL package. Note that since the $m r 1$ and $m r 2$ arrays have been merged into the same memory Memoryl, offsets (MR1OFFSET and MR2OFFSET) must be added to any array addresses; likewise for $t m r 1$ and $t m r 2$, which both reside in Memory2.

There are two important points to note in this example. First, note the large amount of detail that must be added to the specification as a result of creating a system-level design. Presently, designers must manually incorporate this detail, resulting in longer specification times. Moreover, if the system-level design serves as the first captured specification, then we can expect many more functional errors, since the specification writer must consider many detailed issues that detract from a focus on the system's functionality. Second, it is crucial that the designer be given access to these newly introduced details. Many of those details involve important design decisions that the designer must be aware of and must be able to change; for these reasons, generation of a refined specification can be seen as extremely important. After refinement, the functional specification of each component is just that-a specification, not an implementation. This means that for a software component (as well as a hardware component), there may be more than one process in the component's functional specification. These processes will need to be merged into a single control thread, but such merging is part of the implementation task for the component. Thus, the refined specification is a unique and important intermediate representation of functionality, necessary to verify the systemlevel allocation and partitioning decisions we have made, without yet requiring detailed implementation decisions for each component. Further details on refinement can be found in [8] and [46].

\section{EXPERIMENTS}

We have conducted a series of experiments to explore design alternatives for several industrial examples. Here we present results for one particular example: a fuzzy-logic controller [47].

Four library components were available: a standard processor (Intel 8051) and three custom processors with 50, 100, and $150 \mathrm{k}$ gates. Each component had an associated dollar cost. For the experiment, we automatically generated all possible allocation combinations of these components below a certain dollar cost. For each allocation, we partitioned automatically using simulated annealing and a cost function that sought to meet all size and pin constraints while minimizing execution time.

Fig. 16 shows results for the fuzzy-logic controller for 35 different allocations. Allocation 1 consisted of just the 8051 standard processor, and had an execution time of over 150000 $\mathrm{ms}$, so its point is not shown on the graph. Allocation 2 consisted of just one $50 \mathrm{k}$ gate custom processor, but the processor's size constraint was violated so that point is not shown either. Allocation 3 consisted of one 8051 standard processor and one 50-k gate custom processor, resulting in an execution time of $18115 \mathrm{~ms}$. Allocation 4 consisted of just one $100-\mathrm{k}$ gate processor, but again this resulted in a size violation, so the point is not shown. Allocation 5 consisted of one 8051 and one 100-k gate custom processor, yielding an execution time of $7721 \mathrm{~ms}$. Subsequent higher cost allocations yield no better execution time. For example, allocation 31 consisted of one 8051, one 50-k custom processor, one 100-k custom processor, and two 150-k custom processors, and yielded an execution time of $9785 \mathrm{~ms}$. Conceptually, we should have been able to achieve $7721 \mathrm{~ms}$ by just using the 100-k custom processor, but the simulated annealing formulation simply did not find a solution using just that custom processor along with the 8051; instead, functions were assigned to multiple custom processors, requiring interprocessor communication and hence the longer execution time.

SpecSyn thus aids the designer to get a feel for the design space, enabling him to focus on promising points. The above data was generated automatically in $1 \mathrm{~h}$ running on a Sparc 2. There are numerous other types of tradeoffs that can also be generated.

SpecSyn was used by an industry engineer to design the fuzzy-logic controller. The partitioning results obtained matched favorably with those obtained by another engineer who did a manual partition. The system-level design obtained by SpecSyn consisted of 5 FPGA's. Each was implemented using high-level synthesis, and NeoCAD tools were used to complete the design. Details of this experiment can be found in [47]. We summarize them briefly in Fig. 17. The SpecCharts language was used for the initial specification. Note the reduction in the number of lines when using SpecCharts as opposed to VHDL for the specification (see [8] for other experiments which demonstrate the reduction in specification time, specification errors, comprehension time, and lines of code). Also note the large increase in the number of lines for the refined specification; since this is automatically generated, the designer is relieved from the tedious effort of having to write the refined specification himself. Finally, note the very large size of the VHDL after its structural implementation; such a large amount of 


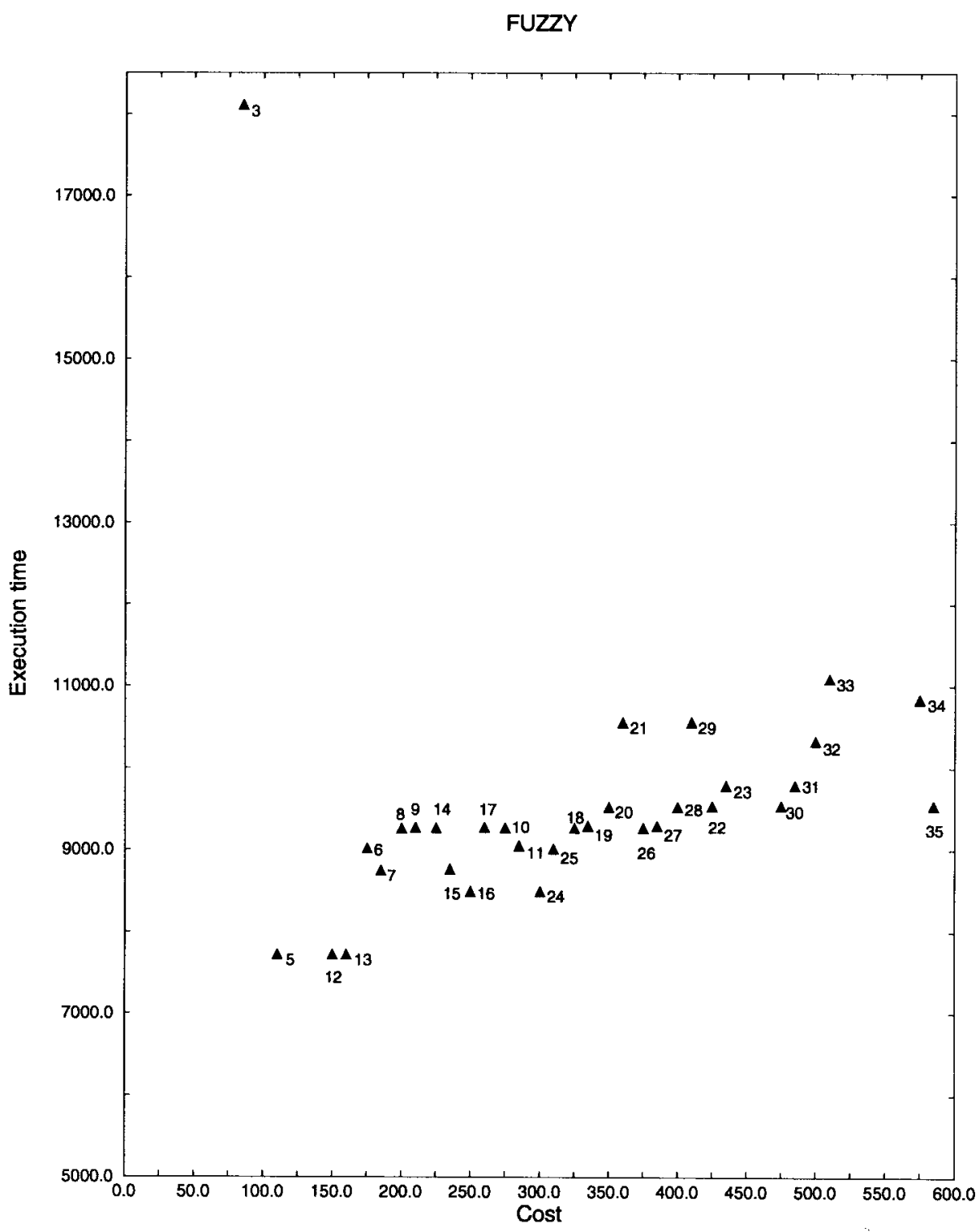

Fig. 16. Exploration for the fuzzy-logic controller.

information is very difficult to work with, so starting with a functional specification enables a tremendous increase in designer comprehension. The entire implementation was obtained in roughly 100 man-hours with the aid of SpecSyn and high-level synthesis, which is nearly a ten times reduction in design time from the six months required to obtain the design manually.

\section{Related Work, Current Status, AND Future Work}

Several other system-level design environments have also evolved. TOSCA [48], [49] focuses on control-dominated systems. A hierarchical FSM input is converted to a process algebra internal format based on a CSP-like model, which is partitioned among an architecture consisting of a standard processor, memory, system bus, and some number of custom processors that can share local buses. Partitioning is performed manually or using a hierarchical clustering heuristic,

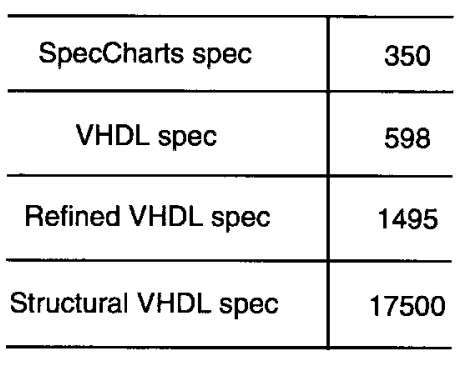

Fig. 17. Fuzzy-logic controller industry design summary.

incorporating formal transformations such as parallelization. Several metrics guide the partitioning. Processes partitioned to software are output in a virtual instruction set (VIS), which is later translated for a particular processor, thus achieving some processor independence. Synthesis is applied to the output and the results used to guide further iterations. The VIS is similar 
to SpecSyn's generic instruction set, except that SpecSyn only uses the set for estimation purposes; SpecSyn outputs software at the algorithmic level, in accordance with the SER methodology.

COSYMA [12] focuses on microcontroller-based systems. An extended $\mathrm{C}$ input is converted to a basic-block and statement-level graph, which is partitioned among an architecture consisting of a standard processor, custom processor, memory and bus. Fast indirect metrics guide the simulated annealing partitioning, the resulting implementation is then analyzed using more complex metrics, and the results are used to guide further iterations. Vulcan II [13] uses a similar architecture and applies a greedy partitioning heuristic with fast indirect metrics. Recent focus has been on analyzing input constraints for use during partitioning and synthesis.

A large number of other approaches exist. Summaries can be found in [29] and [50].

SpecSyn possesses many unique features. First, SpecSyn outputs a system-level description in order to support the SER methodology. Second, SpecSyn is intended to support a variety of system architectures, heuristics, estimation models, and cost functions; no one version of any of these items is advocated for all possible systems. For example, a suite of heuristics is provided, with easy ability to add new ones. Third, SpecSyn uses a two-level estimation method in which considerable effort is spent on both preestimation and online-estimation.

SpecSyn currently consists of over 150000 lines of C code, and has been under development since 1989. Its main interface consists of a spreadsheet-like display showing each component and functional object along with annotations, constraints and metric values for each. Menu options permit designers to perform any of a number of design tasks, whose results are then reflected by updating values in the display; violated constraints are flagged for the user. SpecSyn has been released to several universities and to over 20 companies, and experiments with industry examples are ongoing.

Some limitations lend themselves to future work. First, SpecSyn does not currently support scheduling of the coarsegrained behaviors on the processors to which they are assigned, since in manual design, the system-level allocation and partition decisions are usually made before such scheduling decisions. However, in an automated approach, such scheduling might prove useful. Second, SpecSyn does not currently incorporate the postsynthesis metric values back into subsequent explore/refine iterations. Such incorporation could prove very useful. Third, a method should be introduced to allow designers to provide manual metric estimations. Such a method could be as simple as accepting numbers for use during preestimation, or as complex as using designer-defined expressions for combining annotations during online-estimation. Fourth, a method for design from partial specifications should be implemented. The method for allowing manual metric estimations would likely form a large part of this method. Fifth, estimation models must be continually improved to account for additional architectural features, such as pipelining, caching, and real-time operating systems, and to model fixed-processors like DMA controllers, Fourier transform blocks, Ethernet controllers, MPEG de- coders, etc. Some work on pipelining has been reported in [51] and [52]. Sixth, exploration might be improved by considering ranges of designs during partitioning, rather than a single point in the range as is currently done. Seventh, transformations, such as parallelization, need to be developed and integrated with partitioning, as they play a key role in enabling good final implementations. Eighth, as package borders continually change and more components find their way onto a chip, a general method of partitioning and estimating for hierarchical components needs to be developed. Finally, a variety of input languages, such as C, Statecharts, and synchronous-dataflow-based languages, need to be supported.

\section{CONCLUSIONS}

We have introduced a specify-explore-refine paradigm for system design. Our specification technique focuses on understandable specifications, which in turn encourages the use of front-end languages such as SpecCharts. Our approach to exploration uses preestimation and online-estimation to achieve both fast and accurate estimates, supports a variety of partitioning heuristics, and is intended to be continually extended, enabling a designer to examine numerous alternative designs quickly. Our refinement techniques automatically insert details into the specification that would otherwise have been manually written by the designer, thus relieving the designer of tedious effort. We expect that this paradigm and tool will eventually result in a 100-h design cycle, and our experiments demonstrate the feasibility of such a dramatic reduction in design time from current practice.

\section{REFERENCES}

[1] D. D. Gajski, N. D. Dutt, C. H. Wu, and Y. L. Lin, High-Level Synthesis: Introduction to Chip and System Design. Boston, MA: KluwerAcademic, 1991.

[2] J. Vanhoof, K. VanRompaey, I. Bolsens, and H. DeMan, High-level Synthesis for Real-Time Digital Signal Processing. Boston, MA: KluwerAcademic, 1993.

[3] G. DeMicheli, A. Sangiovanni-Vincentelli, and P. Antognetti, Design Systems for VLSI Circuits: Logic Synthesis and Silicon Compilation. Amsterdam, The Netherlands: Martinus Nijhoff, 1987.

[4] C. A. R. Hoare, "Communicating sequential processes," Commun. ACM, vol. 21, no. 8, pp. 666-677, 1978.

[5] D. Harel, H. Lachover, A. Naamad, A. Pnueli, M. Politi, R. Sherman, and A. Shtul-Trauring, "STATEMATE: A working environment for the development of complex reactive systems," in Proc. Int. Conf. Software Eng., 1988, pp. 396-406.

[6] F. Vahid, S. Narayan, and D. Gajski, "SpecCharts: A VHDL front-end for embedded systems," IEEE Trans. Comput., pp. 694-706, 1995

[7] E. Lee and D. Messerschmitt, "Synchronous data flow," Proc. IEEE vol. 75 , pp. 1235-1245, Sept. 1987.

[8] D. D. Gajski, F. Vahid, S. Narayan, and J. Gong, Specification and Design of Embedded Systems. Englewood Cliffs, NJ: Prentice-Hall, 1994.

[9] F. Vahid, "Procedure exlining: A transformation for improved system and behavioral synthesis," in Proc. Int. Symp. Syst. Synthesis, 1995, pp. 84-89.

[10] _ _ "Procedure cloning: A transformation for improved system-level functional partitioning," in Proc. European Design Test Conf. (EDTC), 1997, pp. 487-492.

[11] J. Gong, D. Gajski, and S. Narayan, "Software estimation using a generic processor model," in Proc. European Design Test Conf. (EDTC), 1995, pp. 498-502.

[12] R. Ernst, J. Henkel, and T. Benner, "Hardware-software cosynthesis for microcontrollers," IEEE Design Test Comput., pp. 64-75, Dec. 1994. 
[13] R. Gupta and G. DeMicheli, "Hardware-software cosynthesis for digital systems," IEEE Design Test Comput., pp. 29-41, Oct. 1993.

[14] M. C. McFarland and T. J. Kowalski, "Incorporating bottom-up design into hardware synthesis," IEEE Trans. Comput., pp. 938-950, Sept. 1990.

[15] E. D. Lagnese and D. E. Thomas, "Architectural partitioning for system level synthesis of integrated circuits," IEEE Trans. Comput., vol. 10, pp. 847-860, July 1991

[16] C. H. Gebotys, "An optimization approach to the synthesis of multichip architectures," IEEE Trans. VLSI Syst., vol. 2, pp. 11-20, Mar. 1994.

[17] Y. Y. Chen, Y. C. Hsu, and C. T. King, "MULTIPAR: Behavioral partition for synthesizing multiprocessor architectures," IEEE Trans. VLSI Syst., vol. 2, pp. 21-32, Mar. 1994.

[18] K. Kucukcakar and A. Parker, "CHOP: A constraint-driven system-level partitioner," in Proc. Design Automation Conf., 1991, pp. 514-519.

[19] R. Gupta and G. DeMicheli, "Partitioning of functional models of synchronous digital systems," in Proc. Int. Conf. Computer-Aided Design, 1990, pp. 216-219.

[20] P. Eles, Z. Peng, K. Kuchcinski, and A. Doboli, "Hardware-software partitioning with iterative improvement heuristics," in Proc. Int. Symp. Syst. Synthesis, 1996, pp. 71-76.

[21] F. Vahid and D. Gajski, "Specification partitioning for system design," in Proc. Design Automation Conf., 1992, pp. 219-224.

[22] F. Vahid, T. D. M. Le, and Y. C. Hsu, "A comparison of functional and structural partitioning," in Proc. Int. Symp. Syst. Synthesis, 1996, pp. $121-126$

[23] X. Xiong, E. Barros, and W. Rosentiel, "A method for partitioning UNITY language in hardware and software," in Proc. European Design Automation Conf. (EuroDAC), 1994.

[24] F. Vahid, J. Gong, and D. D. Gajski, "A binary-constraint search algorithm for minimizing hardware during hardware-software partitioning," in Proc. European Design Automation Conf. (EuroDAC), 1994, pp. 214-219.

[25] P. Eles, Z. Peng, and A. Doboli, "VHDL system-level specification and partitioning in a hardware/software co-synthesis environment," in Proc. Int. Workshop on Hardware-Software Co-Design, 1992, pp. 49-55.

[26] A. Kalavade and E. A. Lee, "A global criticality/local phase driven algorithm for the constrained hardware/software partitioning problem," in Proc. Int. Workshop on Hardware-Software Co-Design, 1994, pp. $42-48$.

[27] J. G. D'Ambrosio and X. Hu, "Configuration-level hardware/software partitioning for real-time embedded systems," in Proc. Int. Workshop Hardware-Software Co-Design, 1994, pp. 34-41.

[28] F. Vahid and D. D. Gajski, "Clustering for improved system-level functional partitioning," in Proc. Int. Symp. Syst. Synthesis, 1995, pp. 28-33.

[29] D. D. Gajski and F. Vahid, "Specification and design of embedded hardware-software systems," IEEE Design Test Comput., vol. 12, pp. 53-67, 1995.

[30] J. V. Rajan and D. E. Thomas, "Synthesis by delayed binding of decisions," in Proc. Design Automation Conf., 1985.

[31] F. Vahid and D. Gajski, "Incremental hardware estimation during hardware/software functional partitioning," IEEE Trans. VLSI Syst., vol 3, pp. 459-464, Sept. 1995.

[32] J. W. Hagerman and D. E. Thomas, "Process transformation for system level synthesis," Tech. Rep. CMUCAD-93-08, 1993.

[33] R. A. Walker and D. E. Thomas, "Behavioral transformation for algorithmic level IC design," IEEE Trans. Comput., pp. 1115-1128, Oct. 1989.

[34] A. Nicolau and R. Potasman, "Incremental tree height reduction for high level synthesis," in Proc. Design Automation Conf., 1991, pp. 770-774.

[35] M. Girkar and C. D. Polychronopoulos, "Automatic extraction of functional parallelism from ordinary programs," IEEE Trans. Parallel Distrib. Syst., pp. 166-178, 1992.

[36] F. Vahid, "Port calling: A transformation for reducing I/O during multipackage functional partitioning," in Int. Symp. Syst. Synthesis, 1997.

[37] S. Narayan and D. D. Gajski, "Synthesis of system-level bus interfaces," in Proc. European Conf. Design Automation (EDAC), 1994.

[38] _ "Protocol generation for communication channels," in Proc. Design Automation Conf., 1994, pp. 547-551.

[39] G. Borriello and R. H. Katz, "Synthesis and optimization of interface transducer logic," in Proc. Int. Conf. Computer-Aided Design, 1987.

[40] J. Akella and K. McMillan, "Synthesizing converters between finite state protocols," in Proc. Int. Conf. Computer Design, 1991.

[41] J. S. Sun and R. W. Brodersen, "Design of system interface modules," in Proc. Int. Conf. Computer-Aided Design, 1992, pp. 478-481.

[42] D. Becker, R. K. Singh, and S. G. Tell, "An engineering environment for hardware/software co-simulation," in Proc. Design Automation Conf., 1992, pp. 129-134.
[43] R. Gupta, C. N. Coelho, and G. DeMicheli, "Synthesis and simulation of digital systems containing interacting hardware and software components," in Proc. Design Automation Conf., 1992, pp. 225-230.

[44] A. Kalavade and E. A. Lee, "A hardware/software codesign methodology for DSP applications," IEEE Design Test Comput., 1993.

[45] S. Sutarwala and P. Paulin, "Flexible modeling environment for embedded systems design," in Proc. Int. Workshop Hardware-Software Co-Design, 1994, pp. 124-130.

[46] J. Gong, D. Gajski, and S. Bakshi, "Model refinement for hardwaresoftware codesign," in Proc. European Design Test Conf. (EDTC), 1996.

[47] L. Ramachandran, D. D. Gajski, S. Narayan, F. Vahid, and P. Fung, "Toward achieving a 100-hour design cycle: A test case," in Proc. European Design Automation Conf. (EuroDAC), 1994, pp. 144-149.

[48] A. Balboni, W. Fornaciari, and D. Sciuto, "Partitioning and exploration strategies in the TOSCA co-design flow," in Proc. Int. Workshop Hardware-Software Co-Design, 1993, pp. 62-69.

[49] S. Antoniazzi, A. Balboni, W. Fornaciari, and D. Sciuto, "A methodology for control-dominated systems codesign," in Proc. Int. Workshop Hardware-Software Co-Design, 1994, pp. 2-9.

[50] W. H. Wolf, "Hardware-software co-design of embedded systems," Proc. IEEE, vol. 82, pp. 967-989, July 1994.

[51] S. Bakshi and D. D. Gajski, "A component selection algorithm for highperformance pipelines," in Proc. European Design Automation Conf. (EuroDAC), 1994, pp. 400-405.

[52] S. Bakshi and D. D. Gajski, "A memory selection algorithm for highperformance pipelines," in Proc. European Design Automation Conf. (EuroDAC), 1994, pp. 124-129.

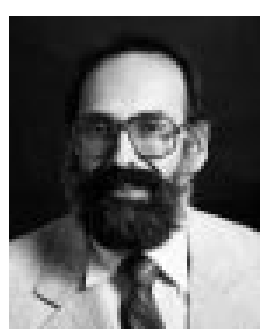

Daniel D. Gajski (M'77-SM'83-F'94) received the Dipl.Ing. and M.S. degrees in electrical engineering from the University of Zagreb, Croatia, and the $\mathrm{Ph} . \mathrm{D}$. degree in computer and information sciences from the University of Pennsylvania, Philadelphia.

After ten years of industrial experience in digital circuits, switching systems, supercomputer design, and VLSI structures, he spent ten years in academia with the Department of Computer Science at the University of Illinois, Urbana-Champaign. Presently, he is a Professor in the Department of Information and Computer Sciences at the University of California, Irvine. His interests are in multiprocessor architectures and science of design. He is editor of the book High-Level Synthesis: An Introduction to Chip and System Design (New York: Kluwer-Academic, 1992) and Specification and Design of Embedded Systems (Englewood Cliffs, NJ: Prentice-Hall, 1994), and the author of Principles of Digital Design (Englewood Cliffs, NJ: Prentice-Hall, 1985).

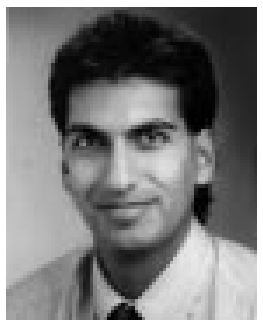

Frank Vahid (S'89-M'93) received the B.S. degree in electrical and computer engineering from the University of Illinois, Urbana-Champaign, in 1988. $\mathrm{He}$ received the M.S. and Ph.D. degrees in computer science from the University of California, Irvine, in 1990 and 1994, respectively, where he was an SRC fellow.

He has worked as an Engineer at Hewlett-Packard and AMCC. He is currently an Assistant Professor in the Department of Computer Science at the University of California, Riverside. His research in terests include hardware/software codesign of embedded systems, intellectual property development and use, and functional partitioning. He is coauthor of the book Specification and Design of Embedded Systems.

Dr. Vahid served as Program Chair for the International Symposium on System Synthesis in 1996 and as General Chair in 1997. 


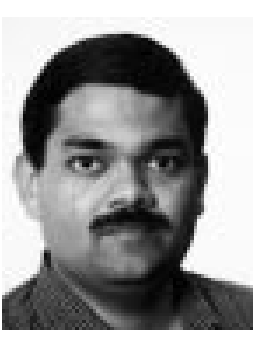

Sanjiv Narayan received the B.S. degree in computer science from the Indian Institute of Technology, New Delhi, in 1988. He received the M.S. and $\mathrm{Ph} . \mathrm{D}$. degrees in computer science as a Chancellor's Fellow at the University of California, Irvine, in 1990 and 1994, respectively.

He is currently with Ambit Design Systems, Santa Clara, CA, where he is associated with the research and development of behavioral synthesis tools. His current research interests include behavioral synthesis, system specification and modeling, and interface synthesis. He is also a coauthor of Specification and Design of Embedded Systems (Englewood Cliffs, NJ: Prentice-Hall, 1994).

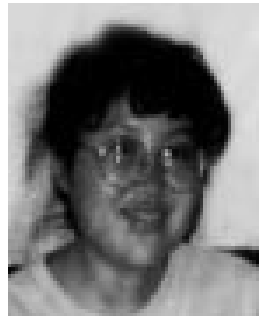

Jie Gong received the M.S. and Ph.D. degrees in computer science from the University of California, Irvine. She received the B.S. degree in computer engineering from the Tsinghua University, Beijing, People's Republic of China.

She was working at the Unified Design System Laboratory of Motorola, Inc., and currenlty she is with Qualcomm, Inc. Her research interests include behavioral synthesis and system-level design. She is a coauthor of the book, Specification and Design of Embedded Systems (Englewood Cliffs, NJ: Prentice-

Dr. Gong is a member of the ACM. 